\title{
The role of macrophages in tumour immunity
}

\author{
PETER ALEXANDER \\ From the Chester Beatty Research Institute, Sutton, Surrey
}

Immunologically specific rejection of syngeneic tumours by experimental animals which have been immunized is well established, and there are many reports which show that the immune state can be transferred by lymphoid cells. The nature of the effector mechanisms is complex and not only may vary from one tumour system to another but also may depend on the anatomical site of tumour implantation. The role of macrophages in rejection reactions, particularly allografts, was remarked upon by Gorer (1956) and later demonstrated with in-vitro techniques by Granger and Weiser (1964). Our attention focused on the role of macrophages in rejection of syngeneic ascitic lymphomas to which immunity can be induced by injection of $x$-irradiated cells but the lymphoid cells from hyperimmunized mice behaved in a seemingly paradoxical manner when mixed with the specific lymphoma cells. When the cell mixtures were injected into normal DBA/2 mice growth of the tumour cells was arrested, but when the mixtures were incubated in culture, the lymphoma cells grew normally in the presence of the lymphoid cells from hyperimmunized mice (Alexander, Connell, and Mikulska, 1966). We were led to the hypothesis that with these particular syngeneic tumours, their injection together with immune lymphoid cells required a cooperation with other and non-immune cells, such as macrophages, to produce rejection (Evans and Alexander, 1970).

\section{The 'Armed' and the 'Activated' Macrophage}

Support for this concept was provided by experiments in which it was shown that macrophages which inhibit the growth of tumour cells in an immunologically specific way in vitro can be obtained in three ways: (1) directly from the peritoneal cavity of hyperimmunized mice (Evans and Alexander, 1972a); (2) by incubation of non-immune macrophages with spleen cells from hyperimmune mice (Evans and Alexander, 1970, 1972a); (3) by treatment of nonimmune macrophages with a factor called 'specific macrophage arming factor' (SMAF) which is produced by immune T-cells after they have been incubated for some hours with the specific antigen (Evans and Alexander, 1971, 1972b; Evans, Grant,
Cox, Steele, and Alexander, 1972). The term 'arming' is used to describe the process whereby macrophages acquire in vitro the capacity to inhibit the growth of and kill target cells in an immunologically specific manner. Lymphoid cells from singly immunized mice failed to arm macrophages, even though the mice were strongly immune to challenge with dividing lymphoma cells, and peritoneal macrophages from these mice were only weakly cytotoxic. However, the same lymphoid cells when exposed to the specific lymphoma cells released a factor which was able to arm normal macrophages suggesting that singly immunized mice required a second exposure to antigen before immune peritoneal macrophages and arming lymphoid cells could be demonstrated.

The growth inhibitory action of macrophages from suitably immunized animals or 'armed' in vitro can be directed against the normal transplantation antigen of the tumour cells or in syngeneic systems against the tumour-specific antigens. Such macrophages exert their action by membrane contact with the target cell. Phagocytosis is a late event which occurs only after the target cell has been killed. Immune or armed macrophages following incubation with the specific antigen undergo a change which we have called 'activation' (Evans and Alexander, $1972 \mathrm{c}$ ), after which they acquire the property to inhibit in vitro the growth of all sarcoma and lymphoma cells tested by us. ${ }^{1}$ This transition from arming to activation is illustrated diagrammatically in figure 1. Table I gives experimental data of this transition, using two antigenically unrelated lymphoma cells, SL2 and TLX9. Macrophages from mice immunized against SL2 only affect the growth of SL2 cells and the same applies to TLX9 cells

\footnotetext{
'Activated macrophages whether produced by specific interaction with antigen or by treatment with double-stranded RNA or endotoxin (Alexander and Evans, 1971) inhibit the growth in vitro of lymphoma and sarcoma cells but have no effect on the growth of rapidly dividing embryo fibroblasts. Remington's group reported that macrophages obtained from mice infected with Toxoplasma inhibited the growth of the long-established line of mouse fibroblasts (L-cells) which can be considered to be malignant cells but had no effect on the growth of mouse embryo cells (Hibbs, Lambert, and Remington, 1972a). However, when after prolonged culture the embryo cells developed an abnormal growth pattern then they became susceptible to inhibition by activated macrophages (Hibbs, Lambert, and Remington, 1972b).
} 


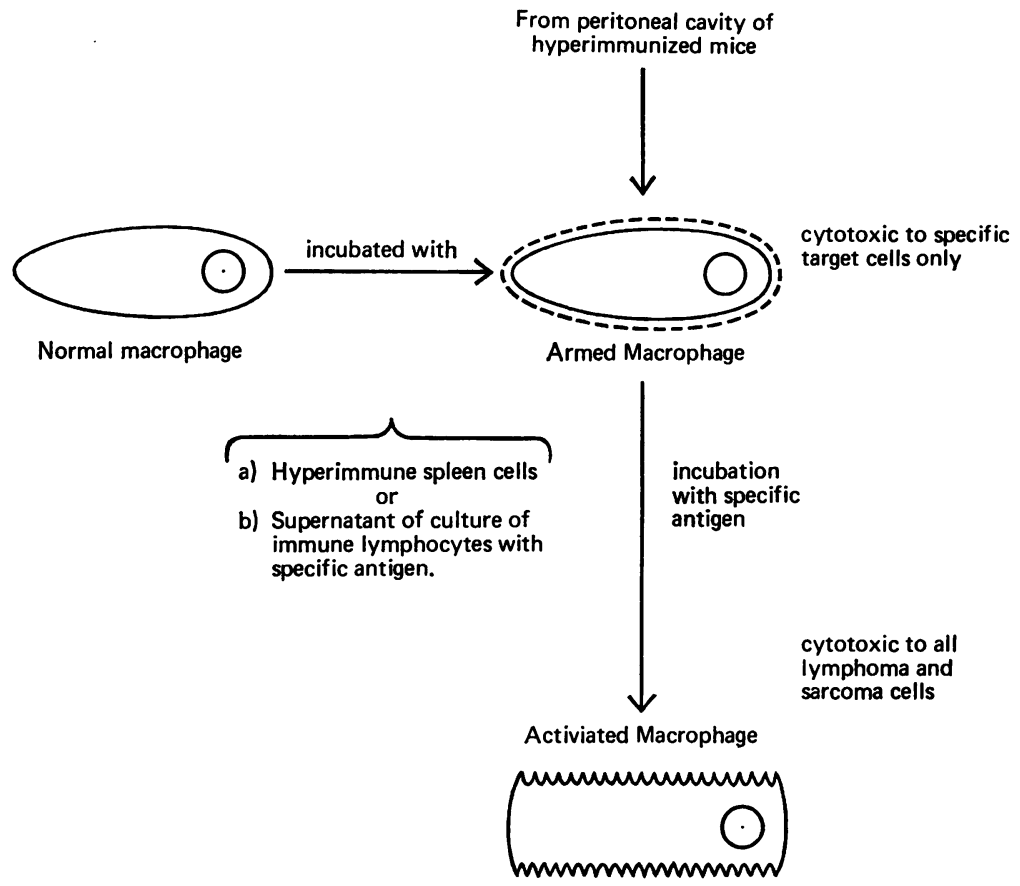

Fig 1 Mechanism of macrophage cytotoxicity.

However, when anti-SL2 macrophages are incubated with SL2 cells, they then acquire the capacity to kill TLX9 cells as well and again the reverse situation applies to the TLX9. This transition to the activated state, however, requires the specific antigen. When anti-SL2 macrophages are incubated with TLX9 they do not become activated and will only kill SL2 cells.

Of relevance to the anti-tumour action by stimulation of the reticuloendothelial system are experiments with macrophages from mice immunized with BCG. Macrophages taken from mice which have been injected with BCG two weeks earlier are not cyto- toxic to tumour cells, but when such macrophages are incubated for four hours with the soluble antigen from tubercle organisms (PPD) they acquire the capacity to kill lymphoma cells (Evans and Alexander, 1972c). Transformation from an armed to an activated state by contact with specific antigen also occurs in vivo (Evans and Alexander, 1972c; Alexander, Evans, and Grant, 1972). Figure 2 shows that when mice are immunized three times with SL2 cells and the macrophages taken 10 days after the last immunization they are specifically cytotoxic, ie, they affect SL2 cells but not TLX9 cells. However, if they are taken between onc to

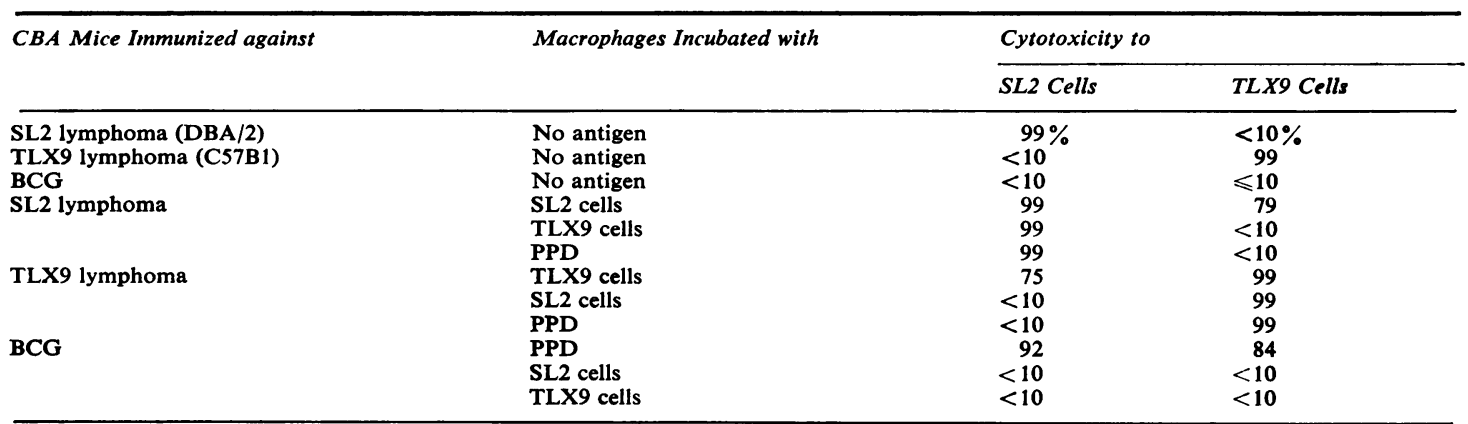

Table I Cytotoxicity of macrophages from immunized mice 
Specificity of Peritoneal Macrophages *

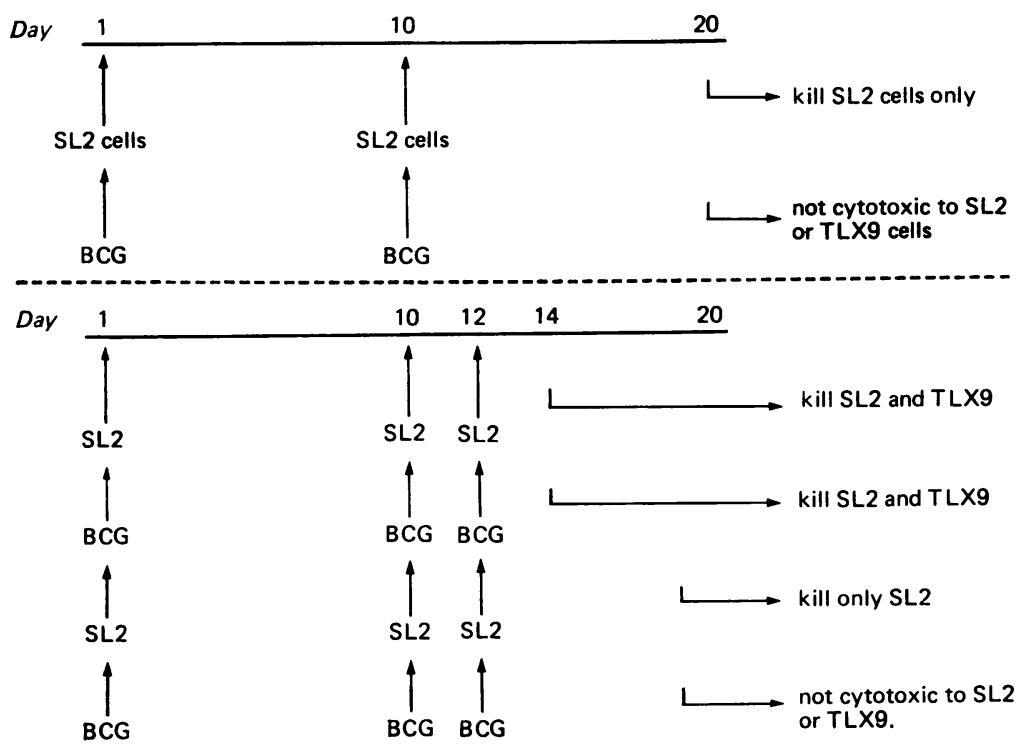

Fig 2 Immunological specificity of macrophages from $C 57 / B 1$ mice immunized with live SL2 cells (DBA/2 genotype) or BCG.

*Removed either on day 14 or day 20 as indicated

three days after the last injection of SL2 cells, they are activated and inhibit the growth of both SL2 and TLX9 cells. Presumably macrophages after two immunizations were armed and the final injection of SL2 cells caused them to become activated in vivo but in the absence of further antigen they lose activation. The same pattern is seen with BCG. If the macrophages are taken more than 10 days after the last BCG injection they have no effect on tumour cells. However, if taken within three days of the last inoculation of BCG, they are activated and inhibit the growth of lymphoma and sarcoma cells in vitro.

Activated macrophages are found in mice with a persisting infection without deliberately applying a second antigenic challenge. The infection causes macrophages to be armed and the persistence of the infective agent ensures that the immune macrophages become and remain activated. This phenomenon was clearly demonstrated by Hibbs et al (1972a) who found that following infection with Toxoplasma, peritoneal macrophages of mice with active toxoplasmosis were cytotoxic to tumour cells in vitro, ie, these macrophages were activated. These workers also showed that mice with activated macrophages due to toxoplasmosis were more resistant to challenge with tumour cells (Hibbs, Lambert, and Remington, 1972c) and to viral carcinogenesis (Hibbs, Lambert, and Remington, 1972d). We made the same observavation by accident (Evans and Alexander, 1972c): our mouse colony became infected with Pasteurella which persisted in the mice for months; the macrophages from such mice were non-specifically cytotoxic. Macrophages can also be 'activated', ie, inhibit the growth of sarcoma and lymphoma cells in an immunologically non-specific way by exposure, in vitro or in vivo, to very low concentrations of endotoxin or double-stranded RNA (Alexander and Evans, 1971) (see fig 3). Such macrophages which have been 'activated' pharmacologically cannot be distinguished in their growth-inhibitory properties from macrophages that have been 'immunologically' activated.

\section{The Role of Macrophages in Host Resistance to Tumours}

Gorer (1956) felt that macrophages were involved in the rejection of tumour cells injected intraperitoneally into immunized mice from studies of the cells present 


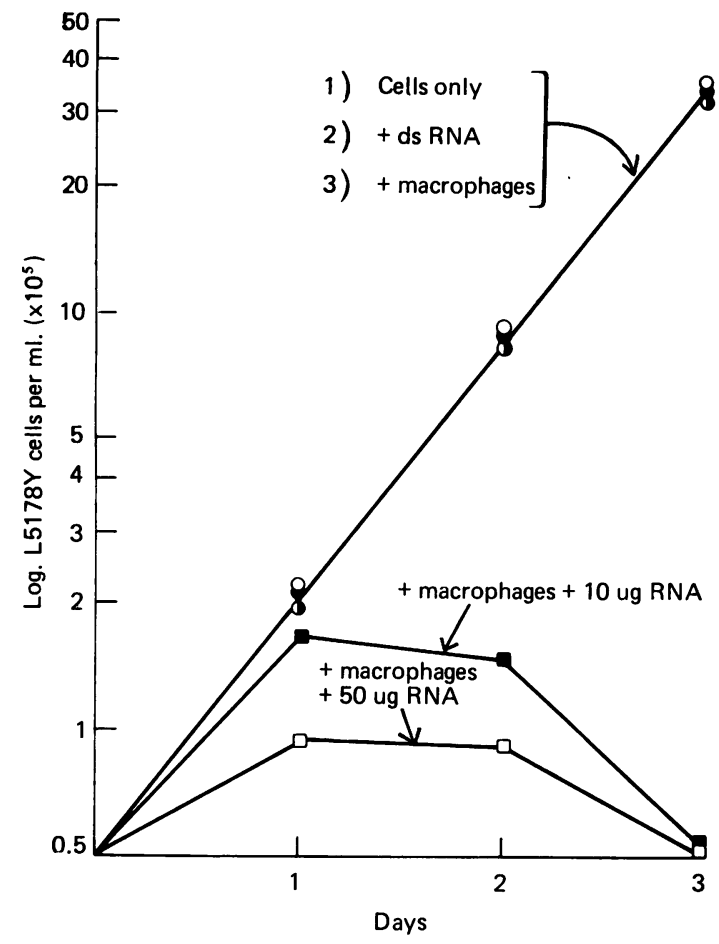

Fig 3 The inhibition of growth in vitro of lymphoma cells by a monolayer of peritoneal macrophages that had been activated by treatment with double strand RNA (ds RNA).

The top curve shows that the lymphoma cells grow at the normal rate in the presence of either ds RNA or normal macrophages. The bottom curves show that the addition of ds RNA to the monolayer of macrophages renders this growth inhibitory to the lymphoma cells.

in the peritoneal cavity. That macrophages may also be involved in the reaction of the syngeneic host to solid tumours is indicated by the finding of Evans (1972) that sarcomas in mice and rats may contain more than $50 \%$ of macrophages. These macrophages are difficult to recognize histologically and the identification of cells in a tumour as macrophages relied on testing the cells from a tumour after they had been brought into suspension by trypsinization.

The criteria for identification and characterization of tumour macrophages were (1) rapid, ie, within five minutes at $37^{\circ} \mathrm{C}$, adherence and spreading either in the haemocytometer or in culture vessels. After fixation in methanol and staining these cells were typically monocytic and polymorphs, when present, never exceeded $10 \%$ of the total macrophage population; (2) resistance to detachment by trypsini- zation; (3) the ability to phagocytose colloidal carbon rapidly; (4) the ability to phagocytose the murine lymphoma cells in the presence of alloantiserum; (5) lysis by rabbit antimouse macrophage serum (AMS) in the presence of complement.

Evans (1972) showed that the macrophages in the tumour were derived from the host. Tumours were produced by inoculation of $(a)$ a cell suspension derived from the tumour and containing both tumour cells and macrophages, and of $(b)$ a cell suspension from which the macrophages had been eliminated by treatment in vitro with anti-macrophage serum. By 14 days the macrophage content of the tumours derived from an inoculum of tumour cells without macrophages was the same as those which had grown from cell inoculum containing $30 \%$ of macrophages.

Macrophages may also be involved in the phenomenon of concomitant immunity (Alexander, Evans, and Mikulska, 1973), ie, the situation in which an animal with a growing tumour is able to reject a second inoculum of the same tumour at a distant site in spite of the fact that the primary tumour is growing relentlessly. The relevance of concomitant immunity to metastatic spread is obvious. Figure 4 shows the results from an experiment in which DBA/2 mice were inoculated subcutaneously with graded doses of syngeneic lymphoma cells and were then injected two weeks later with $10^{3}$ lymphoma cells administered intraperitoneally. This intraperitoneal challenge, which gives rise to an ascites tumour in $100 \%$ of untreated normal mice and kills them within 24 days, did not grow in any of the animals that had previously been injected with $10^{5}$ living tumour cells subcutaneously. The protection of the peritoneal cavity was apparently related to the size of the subcutaneous tumour, since if the intraperitoneal challenge was given only seven days after the inoculation of $10^{5}$ cells subcutaneously-as opposed to 14 days as shown in fig 4-no protection was observed and all of the mice died with an ascitic tumour. The introduction of viable tumour cells intraperitoneally does not hasten the death of animals from their subcutaneous tumour and, indeed, there is some indication that it may slow it.

Tests in vitro showed that the peritoneal cavity of the mice bearing subcutaneously growing lymphomata contained macrophages which inhibited the growth of the lymphoma cells and that the draining nodes contained lymphocytes capable of arming macrophages against the lymphoma cells (Alexander, Evans, and Mikulska, 1973). These data are consistent with the hypothesis that the resistance of the peritoneal cavity to tumour growth in a tumour-bearing animal is mediated by macrophages. 


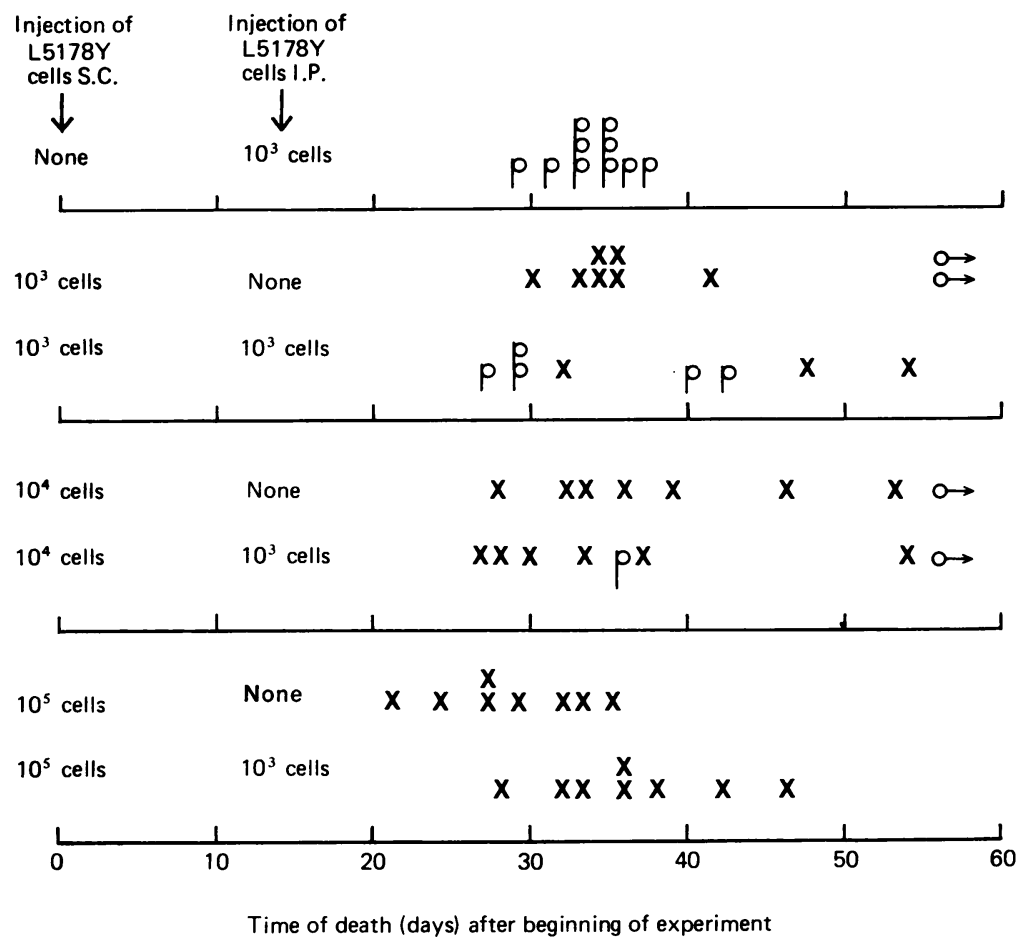

Fig 4 The presence of an established subcutaneous tumour prevents the growth of the same tumour cells injected intraperitoneally 14 days later.

$X$ Represents the time of death of individual mice from a subcutaneous tumour in the absence of any ascites tumour.

$P$ Represents the time of death of mice which at necropsy were shown to carry a large ascites tumour.

o Mice which were free from tumour 55 days after the beginning of the experiment.

\section{Macrophages and 'Immunotherapy'}

The phenomenon of activating macrophages in mice immunized against an antigen totally unrelated to tumours, such as BCG or sheep red cells, can be exploited for immunotherapy. This is illustrated in a series of experiments summarized in table II. Mice were pretreated with BCG and then 12 days later were challenged intraperitoneally with lymphoma cells. The lymphoma cells grew almost as well in the BCG-pretreated as in normal mice (ie, the protective action of non-specific stimulation of the reticuloendothelial system is minimal in this situation). However, PPD injected 24 hours after intraperitoneal inoculation of the tumour cells produced a marked antitumour effect in the BCGpretreated mice. We interpret this antitumour action as being due to the activation of peritoneal macrophages armed against BCG. However, this effect is strictly local. If the PPD is injected intravenously or subcutaneously then there is only a very small effect on the intraperitoneal tumour. Also, if the tumour is grown subcutaneously then intraperitoneal injection of PPD is without effect. It is, however,

\begin{tabular}{|c|c|c|c|c|c|c|}
\hline \multicolumn{4}{|c|}{ Treatment } & \multicolumn{3}{|c|}{$\begin{array}{l}\text { Percentage Mice Surviving after Injection of } \\
\text { Lymphoma Cells for }\end{array}$} \\
\hline Day 1 & Day 8 & Day 20 & Day 21 & Less than 25 Days & 25 to 50 Days & More than 50 Days \\
\hline Nil & Nil & $\begin{array}{l}10^{4} \mathrm{~L} 5178 \mathrm{Y} \\
\text { cells ip }\end{array}$ & Nil & 90 & 10 & 0 \\
\hline Nil & Nil & 10- L5178Y cells ip & PPD ip & 100 & 0 & 0 \\
\hline BCG & BCG & 10- L5178Y cells ip & $\mathrm{Nil}$ & 60 & 40 & 0 \\
\hline BCG & BCG & 10- L5178Y cells ip & PPD ip & 30 & 20 & 50 \\
\hline BCG & BCG & 10- L5178Y cells ip & PPD iv & 60 & 40 & 0 \\
\hline BCG & BCG & 10- L5178Y cells ip & PPD sc & 40 & 60 & 0 \\
\hline
\end{tabular}

Table II The effect of administering PPD on the growth of lymphoma cells ( $L 5178 Y)$ in mice (DBA/2 strain) previously immunized with BCG (Parr, unpublished data) 


\begin{tabular}{ll}
\hline Treatment & Average Survival (Days) \\
\hline Phosphate-buffered saline only $^{1}$ & $15 \cdot 5$ (range 14-16) \\
Non-immune macrophages $^{1}$ & $16 \cdot 5$ (range 14-17) \\
Macrophages from hyperimmunized mices $^{2}$ & $30 \cdot 5$ (range 25-36) \\
\hline
\end{tabular}

Table III Effect of injecting immune macrophages on the growth of L5178 Y cells in vivo'

${ }^{1}$ Peritoneal macrophages were allowed to adhere to a film of collagen and were brought into suspension by treatment with collagenase. The cell suspension contained less than $0.1 \%$ of lymphoid cells.

'DBA/2 mice immunized twice with irradiated L5178Y cells (as described by Evans and Alexander, 1971).

possible to protect with PPD given intravenously against a tumour challenge injected intravenously into mice sensitized to BCG. This experiment may be an example of the phenomenon in man described earlier by Klein (1969). He found that some skin tumours regress following painting or injection of an antigen to which the patient had been sensitized, eg, DNCB painting in DNCB-sensitized subjects or PPD injection into tumours of tuberculin-sensitive subjects. It must be stressed that the practical usefulness of all such treatments is limited because macrophage activation is largely confined to the site (or the node draining the site) into which the antigen had been injected. Hence, this procedure, as Klein has found, can only be used for the eradication of accessible tumours.

An antitumour action in vivo could also be demonstrated by transferring specifically growth-inhibitory macrophages to mice bearing an ascites tumour (Alexander, Evans, and Mikulska, 1973). Peritoneal cells were removed from mice that had been immunized twice with irradiated L5178Y cells and were then grown for $\mathbf{4 8}$ hours on sheets of collagen prepared from rat tails. These cultures were then washed extensively and the cells detached by incubation with collagenase. The cell suspension obtained was totally free from lymphocytes. Table III shows that injection of immune macrophages two days after an intraperitoneal inoculation of $10^{3}$ lymphoma (L5178Y) cells reduced the rate of growth of the tumour in vivo. Since an inoculum of 10 cells kills DBA/2 mice in 35 days we may deduce that the immune macrophages killed about $99 \%$ of the $10^{4}$ tumour cells that are present in the peritoneal cavity two days after the challenge with $10^{3}$ cells. Nonimmune macrophages prepared in the same way did not influence the growth of this tumour.

\footnotetext{
References

Alexander, P., Connell, D. I., and Mikulska, Z. B. (1966t). Treament of a murine leukemia with spleen cells or sera from allogeneic
}

mice immunized against the tumor. Cancer Res., 26, 1508-1515 Alexander, P., and Evans, R. (1971). Endotoxin and double stranded RNA render macrophages cytotoxic. Nature [new Biol.], 232, 76-78.

Alexander, P., Evans, R., and Grant, C. K. (1972). The interplay of lymphoid cells and macrophages in tumour immunity. Ann. Inst. Pasteur, 122, 645-658.

Alexander, P., Evans, R., and Mikulska, Z. B. (1973). Relationship between concomitant immunity and metastasis-the role of macrophages in concomitant immunity involving the peritonea cavity. In Chemotherapy of Cancer Dissemination and Metastasis, edited by S. Garratini and G. Franchi, pp. 177-185. Raven Press, New York.

Evans, R. (1972). Macrophages in syngeneic animal tumours. Transplantation, 14, 468.

Evans, R., and Alexander, P. (1970). Co-operation of immune lymphoid cells with macrophages in tumour immunity. Nature (Lond.), 228, 620-622.

Evans, R., and Alexander, P. (1971). Rendering macrophages specifically cytotoxic by a factor released from immune lymphoid cells. Transplantation, 12, 227-229.

Evans, R., and Alexander, P. (1972a). Role of macrophages in tumour immunity, I. Co-operation between macrophages and lymphoid cells in syngeneic tumour immunity. Immunology, 23, 615-626.

Evans, R., and Alexander, P. (1972b). Role of macrophages in tumour immunity. II. Involvement of a macrophage cytophilic factor during syngeneic tumour growth inhibition. Immunology, 23, 627-636.

Evans, R., and Alexander, P. (1972c). Mechanism of immunologically specific killing of tumour cells by macrophages. Nature (Lond.), 236, 168-170.

Evans, R., Grant, C. K., Cox, Helen, Steele, K., and Alexander, P. (1972). Thymus-derived lymphocytes produce an immunologically specific macrophage arming factor. J. exp. Med., 136, 1318-1322.

Gorer, P. A. (1956). Some recent work on tumor immunity. Advanc. Cancer Res., 4, 149-186.

Granger, G. A., and Weiser, R. S. (1964). Homograft target cells: specific destruction in vitro by contact interaction with immune macrophages. Science, 145, 1427-1429.

Hibbs, J. B., Jr., Lambert, L. H., Jr., and Remington, J. S. (1972a). Possible role of macrophage mediated non-specific cytotoxicity in tumour resistance. Nature [new Biol.], 235, 48-50.

Hibbs, J. B., Jr., Lambert, L. H., Jr., and Remington, J. S. (1972b). In vitro nonimmunologic destruction of cells with abnormal growth characteristics by adjuvant activated macrophages. Proc. Soc. exp. Biol. (N.Y.), 139, 1049-1052.

Hibbs, J. B., Jr., Lambert, L. H., Jr., and Remington, J. S. (1972c). Adjuvant induced resistance to tumor development in mice. Proc. Soc. exp. Biol. (N.Y.), 139, 1053-1056.

Hibbs, J. B., Jr., Lambert, L. H., Jr., and Remington, J. S. (1972d). Control of carcinogenesis: a possible role for the activated macrophage. Science, 177, 998-1000.

Klein, E. (1969). Hypersensitivity reactions at tumor sites. Cancer Res., 29, 2351-2362. 